\title{
LOCALLY UNIVALENT FUNCTIONS IN THE UNIT DISK
}

\author{
NORBERT STEINMETZ
}

\section{Introduction}

Let $f$ be meromorphic and locally univalent in the unit disk $D$ and denote by

$$
\{f, z\}=\left(f^{\prime \prime} \mid f^{\prime}\right)^{\prime}-\frac{1}{2}\left(f^{\prime \prime} \mid f^{\prime}\right)^{2}=2 q(z)
$$

the Schwarzian derivative of $f$.

A well known theorem of Nehari [7] states that $f$ is univalent in each noneuclidean disk of radius $k^{-1 / 2}(k \geqq 1)$ if

$$
|q(z)| \leqq \frac{k}{\left(1-|z|^{2}\right)^{2}} .
$$

Hille [5] has shown that this theorem is sharp.

Later on, Ahlfors and Weill [1] proved that $f$ extends continuously (with respect to the spherical metric) to the boundary, provided $k<1$; in fact, they showed that $f\left(e^{i \theta}\right)$ is Hölder-continuous of order $1-k$. This result was improved by Duren and Lehto [3] who replaced the constant $k$ in (2) by a nondecreasing function $\lambda(|z|), 0<\lambda<1$, and proved that $f$ has a continuous extension to $\partial D$ if $\lambda(r)$ tends "slowly" to 1 :

$$
\frac{1}{1-\lambda(r)}=0\left(\log \frac{1}{1-r}\right) \text { as } r \rightarrow 1-
$$

Recently, Gehring and Pommerenke [4] showed that this remains true even under the original Nehari condition $\lambda(r) \equiv k=1$.

It is well known that $f$ admits the representation

$$
f=\frac{w_{1}}{w_{2}}
$$

where $w_{1}$ and $w_{2}$ are linearly independent solutions of the linear differential equation

$$
w^{\prime \prime}+q(z) w=0 \text {. }
$$


We are mainly concerned with the boundary behaviour of $f$. Since

$$
f^{\prime}=\frac{c}{w_{2}^{2}},
$$

where

$$
-c=\left|\begin{array}{ll}
w_{1} & w_{2} \\
w_{1}^{\prime} & w_{2}^{\prime}
\end{array}\right|
$$

is the Wronskian of $w_{1}$ and $w_{2}$, and

$$
f^{\#}:=\frac{\left|f^{\prime}\right|}{1+|f|^{2}}=\frac{|c|}{\left|w_{1}\right|^{2}+\left|w_{2}\right|^{2}},
$$

we have to estimate the solutions of (5) from below as $|z| \rightarrow 1$.

\section{A monotonicity theorem}

Consider

$$
u(x)=\left|w_{1}\left(x e^{i \theta}\right)\right|^{2}+\left|w_{2}\left(x e^{i \theta}\right)\right|^{2},
$$

$\theta$ fixed, where $w_{1}$ and $w_{2}$ are (not necessarily linearly independent) solutions of (5). Then $u$ is nonnegative in $[0,1)$, and an easy computation gives

$\left(z=x e^{i \theta}\right)$,

$$
u^{\prime}=2 \operatorname{Re}\left[e^{i \theta}\left(w_{1}^{\prime} \bar{w}_{1}+w_{2}^{\prime} \bar{w}_{2}\right)\right]
$$

$$
u^{\prime 2} \leqq 4 u\left(\left|w_{1}^{\prime}\right|^{2}+\left|w_{2}^{\prime}\right|^{2}\right)
$$

by the Cauchy-Schwarz inequality, and

$$
u^{\prime \prime}=2\left(\left|w_{1}^{\prime}\right|^{2}+\left|w_{2}^{\prime}\right|^{2}\right)-2 u \operatorname{Re}\left[e^{2 i \theta} q\left(x e^{i \theta}\right)\right] .
$$

Finally, we get, using the estimate

$$
\operatorname{Re}\left[e^{2 i \theta} q\left(x e^{i \theta}\right)\right] \leqq Q(x)
$$

and (10), (11),

$$
u u^{\prime \prime} \geqq-2 Q(x) u^{2}+\frac{1}{2} u^{\prime 2},
$$

i.e., $u$ is a "supersolution" of the differential equation

$$
y^{\prime \prime}=-2 Q(x) y+\frac{y^{\prime 2}}{2 y} .
$$

It seems quite natural to compare $u$ with "subsolutions" of (14), i.e. solutions of

$$
v v^{\prime \prime} \leqq-2 Q(x) v^{2}+\frac{1}{2} v^{\prime 2} .
$$

However, the usual monotonicity theorems do not apply immediately, since the right hand side of (14) is not quasimonotonically increasing. 
Theorem 1. Let $Q$ be dofined in $(0,1)$ and let $u$ and $v$ be differentiable in $[0,1)$ and twice differentiable in $(0,1)$. Assume further:

I. $u$ is a nonnegative solution of (13) in $0<x<1$;

II. $v$ is a positive solution of (15) in $0<x<1$;

III. $u(0)=v(0)>0$ and $u^{\prime}(0) \geqq v^{\prime}(0)$ or

$u(x)=x^{2}+\alpha x^{3}+o\left(x^{3}\right)$ and $\quad v(x)=x^{2}+\beta x^{3}+o\left(x^{3}\right) \quad$ as $x \rightarrow 0$, where $\alpha \geqq \beta$. Then

$$
u \geqq v \quad \text { in }[0,1) .
$$

Remark. a) It is clear that Theorem 1 remains valid if $(0,1)$ is replaced by an arbitrary interval (of course, III. has to be modified).

b) If we set $U^{\prime}=1 / u, V^{\prime}=1 / v$, inequalities (13) and (15) are equivalent to

$$
\{U, x\} \leqq\{V, x\}
$$

and Theorem 1 may be interpreted as a monotonicity theorem for the Schwarzian derivative: (17) implies $U^{\prime} \leqq V^{\prime}$ if either $U^{\prime}(0)=V^{\prime}(0)>0, U^{\prime \prime}(0) \leqq V^{\prime \prime}(0)$ or else $U^{\prime}(x)=1 / x^{2}-\alpha / x+o(1 / x), V^{\prime}(x)=1 / x^{2}-\beta / x+o(1 / x)$ as $x \rightarrow 0(\alpha \geqq \beta)$.

Proof of Theorem 1. Consider $\omega=u / v$ in the largest interval $\left(0, x_{0}\right)$ containing no zeros of $u$ (if any). A short computation gives

$$
\omega v\left(\omega^{\prime \prime} v+\omega^{\prime} v^{\prime}\right) \geqq \frac{1}{2}\left(\omega^{\prime} v\right)^{2}
$$

and so

$$
\left(\omega^{\prime} v\right)^{\prime} \geqq 0 \quad \text { in } \quad\left(0, x_{0}\right) \text {. }
$$

In both cases III. we have

$$
\omega(0)=1 \text { and } \omega^{\prime}(0) \geqq 0,
$$

implying $\omega^{\prime} v \geqq 0$ in $\left(0, x_{0}\right)$. Therefore, $\omega$ is nondecreasing in $\left(0, x_{0}\right)$ and so $\omega(x) \geqq 1$, or, equivalently,

$$
u(x) \geqq v(x)>0 \quad \text { in } \quad\left(0, x_{0}\right) .
$$

By definition we must have $x_{0}=1$, and Theorem 1 is proved.

\section{Normal functions}

A function $f$ meromorphic in $D$ is called normal if

$$
\sup _{D}\left(1-|z|^{2}\right) f^{\#}(z)<\infty
$$

(see Lehto and Virtanen [6]). In our case $f$ is normal if and only if

$$
\inf _{D} \frac{\left|w_{1}(z)\right|^{2}+\left|w_{2}(z)\right|^{2}}{1-|z|^{2}}>0 .
$$


It is clear how Theorem 1 leads to normality criteria involving $q(z)$. In the simplest case we have $w_{1}(0)=w_{2}^{\prime}(0)=1, w_{1}^{\prime}(0)=w_{2}(0)=0$, and we may apply Theorem 1 to $u(x)=\left|w_{1}\left(x e^{i \theta}\right)\right|^{2}+\left|w_{2}\left(x e^{i \theta}\right)\right|^{2}$ (we have $u(x)=1+O\left(x^{2}\right)$ near $x=0$ ). Since $v(x)=1-x^{2}$ satisfies $v v^{\prime \prime}=-2 /\left(1-x^{2}\right)^{2} v^{2}+1 / 2 v^{\prime 2}$, by Theorem $1 f$ is normal if

$$
\operatorname{Re}\left[e^{2 i \theta} q\left(x e^{i \theta}\right)\right] \leqq \frac{1}{\left(1-x^{2}\right)^{2}}
$$

By Nehari's theorem this is trivial if the real part is replaced by the modulus of $q$.

Theorem 2. Let (12) be satisfied and suppose that, for some $x_{0} \in[0,1)$, the differential inequality $(15)$ has a positive solution in $\left(x_{0}, 1\right)$ such that either

$$
v(x)=1+o\left(x-x_{0}\right)
$$

or else

$$
v(x)=\left(x-x_{0}\right)^{2}+o\left(x-x_{0}\right)^{3}
$$

as $x \rightarrow x_{0}+$. Then there exists a positive constant $C=C\left(x_{0}, f\right)$ such that

Corollary. Suppose

$$
f^{\#}(z) \leqq \frac{C}{v(|z|)} \quad \text { in } \quad x_{0}<|z|<1 .
$$

$$
\operatorname{Re}\left[e^{2 i \theta} q\left(x e^{i \theta}\right)\right] \leqq \frac{1}{\left(1-x^{2}\right)^{2}}+\frac{M}{1-x^{2}},
$$

where $M$ is a constant. Then $f$ is normal in $D$.

Proof of Corollary. It is easily seen that

satisfies

$$
v=\left(x-x_{0}\right)^{2}-\frac{\left(x-x_{0}\right)^{4}}{\left(1-x_{0}\right)^{2}}
$$

in $x_{0} \leqq x<1$, where

$$
v v^{\prime \prime}=-2 Q^{*}(x) v^{2}+\frac{1}{2} v^{\prime 2}
$$

$$
Q^{*}(x)=\frac{1}{\left(1-x^{2}\right)^{2}}\left(\frac{(1+x)\left(1-x_{0}\right)}{1+x-2 x_{0}}\right)^{2}+\frac{2}{1-x^{2}} \cdot \frac{1+x}{1+x-2 x_{0}} .
$$

Since

we have

$$
\frac{1+x}{1+x-2 x_{0}} \geqq \frac{1}{1-x_{0}},
$$

$$
Q^{*}(x) \geqq \frac{1}{\left(1-x^{2}\right)^{2}}+\frac{2}{1-x_{0}} \frac{1}{1-x^{2}},
$$


which is greater than the right hand side of (28) in $x_{0} \leqq x<1$ for $x_{0}$ sufficiently near to 1 . Thus, Theorem 2 gives

$$
f^{\#}(z)=O\left(\frac{1}{v(|z|)}\right)=O\left(\frac{1}{1-|z|^{2}}\right)
$$

as $|z| \rightarrow 1$.

\section{Proof of Theorem 2}

Let $\theta, 0 \leqq \theta<2 \pi$, be fixed and set $z_{0}=x_{0} e^{i \theta}$. If $\left(\stackrel{\circ}{w}_{1}, \stackrel{\circ}{w}_{2}\right)$ is the canonical fundamental system at $z_{0}$ defined by $\stackrel{\circ}{w}_{1}\left(z_{0}\right)=\stackrel{\circ}{w}_{2}^{\prime}\left(z_{0}\right)=1, \stackrel{\circ}{w}_{2}\left(z_{0}\right)=\stackrel{\circ}{w}_{1}^{\prime}\left(z_{0}\right)=0$, we have

$$
\left(\begin{array}{l}
w_{1} \\
w_{2}
\end{array}\right)=\left(\begin{array}{ll}
w_{1}\left(z_{0}\right) & w_{1}^{\prime}\left(z_{0}\right) \\
w_{2}\left(z_{0}\right) & w_{2}^{\prime}\left(z_{0}\right)
\end{array}\right)\left(\begin{array}{c}
\stackrel{\circ}{w}_{1} \\
\stackrel{\leftrightarrow}{w}_{2}
\end{array}\right)
$$

and therefore

$$
\left|w_{1}\right|^{2}+\left|w_{2}\right|^{2} \geqq|c|^{2} K^{-1}\left(\left|\stackrel{\circ}{w}_{1}\right|^{2}+\left|\stackrel{\circ}{w}_{2}\right|^{2}\right),
$$

where $c$ is the Wronskian of $w_{1}$ and $w_{2}$ and

$$
K=\max _{|z|=x_{0}}\left(\left|w_{1}(z)\right|^{2}+\ldots+\left|w_{2}^{\prime}(z)\right|^{2}\right)
$$

is independent of $\theta$. For $u(x)=\left|\stackrel{\circ}{w}_{1}\left(x e^{i \theta}\right)\right|^{2}+\left|\dot{\circ}_{2}\left(x e^{i \theta}\right)\right|^{2}$ or $u(x)=\left|\stackrel{\circ}{w}_{2}\left(x e^{i \theta}\right)\right|^{2}$ we have $u(x)=1+O\left(x-x_{0}\right)^{2}$ or $u(x)=\left(x-x_{0}\right)^{2}+O\left(x-x_{0}\right)^{4}$, respectively (note that $\stackrel{\circ}{2}_{2}^{\prime \prime}\left(z_{0}\right)=0$ ). In both cases Theorem 1 (applied to the interval $\left(x_{0}, 1\right)$ ) gives $u(x) \geqq v(x)$ in $x_{0} \leqq x<1$. Thus, by (7) and (35)

$$
f^{\#}(z) \leqq \frac{K /|c|}{v(|z|)} \quad \text { in } \quad x_{0}<|z|<1 .
$$

\section{Conformal mapping}

Since both the Schwarzian of $f$ and the boundary behaviour of $f$ are invariant under Möbius transforms, we are free to choose special solutions of (1).

We will assume that $w_{1}$ and $w_{2}$ are defined by the initial values

$$
w_{1}(0)=w_{2}^{\prime}(0)=1, \quad w_{2}(0)=w_{1}^{\prime}(0)=0 .
$$

Theorem 3. Let (12) be satisfied and assume that the corresponding differential inequality $(15)$ has a positive solution $v$ in $(0,1)$ such that either

$$
v(x)=x^{2}+o\left(x^{3}\right)
$$

or

$$
v(x)=1+o(x)
$$


as $x \rightarrow 0$, and

$$
\int_{1 / 2}^{1} \frac{d x}{v(x)}<\infty
$$

Then $f$ or $1 / f$, respectively, has boundary values everywhere with modulus of continuity

$$
\Omega(h) \leqq \min _{h \leqq \eta \leqq 1 / 2}\left[\frac{h}{v(1-\eta)}+2 \int_{1-\eta}^{1} \frac{d x}{v(x)}\right] \quad(0<h \leqq 1 / 2) .
$$

Corollary 1. If

$$
\operatorname{Re}\left[e^{2 i \theta} q\left(x e^{i \theta}\right)\right] \leqq \lambda^{2}<\pi^{2}
$$

holds, then $f^{\prime}$ is bounded in $0<\delta \leqq|z|<1$.

Remark. The constant $\lambda$ cannot be replaced by $\pi$, as the example

$$
\{f, z)=2 \pi^{2}
$$

with the special solution

$$
f(z)=-\pi \cot \pi z
$$

shows.

\section{Corollary 2. Suppose}

$$
\operatorname{Re}\left[e^{2 i \theta} q\left(x e^{i \theta}\right)\right] \leqq \frac{\delta(2-\delta)}{\left(1-x^{2}\right)^{2}}+\frac{\delta(1+\delta)}{1-x^{2}},
$$

where $\delta \in(0,1)$ is a constant. Then $f\left(e^{i \theta}\right)$ is Hölder-continuous of order $1-\delta$.

Remark. If (2) holds true $(0<k<1)$, then by Corollary 2 (with $\delta=1-\sqrt{1-k}$ ) $f\left(e^{i \theta}\right)$ is Hölder-continuous of order $\sqrt{1-k}>1-k$. This is the correct order, since one particular solution of

$$
\{f, z\}=\frac{2 k}{\left(1-z^{2}\right)^{2}}
$$

is

$$
f(z)=\left(\frac{1-z}{1+z}\right)^{\varkappa}, \quad x=\sqrt{1-k}
$$

Corollary 3. Let

$$
\operatorname{Re}\left[e^{2 i \theta} q\left(x e^{i \theta}\right)\right] \leqq Q(x)
$$

and assume that the linear differential equation

$$
y^{\prime \prime}+Q(|x|) y=0
$$

possesses a positive solution in $-1<x<1$. Then $f$ has continuous boundary values. Moreover, if

$$
Q(x) \leqq \frac{1}{\left(1-x^{2}\right)^{2}}
$$


then $f\left(e^{i \theta}\right)$ has modulus of continuity

$$
\Omega(h)=O\left(\frac{1}{\log (1 / h)}\right) .
$$

Remark. Recently, Gehring and Pommerenke [4] showed that under the special Nehari condition

$$
|q(z)| \leqq \frac{1}{\left(1-|z|^{2}\right)^{2}}
$$

$f$ maps the unit disk onto a Jordan domain, whose boundary curve has modulus of continuity $O(1 / \log (1 / h))$, or onto the image of a parallel strip under a Möbius transform. I am greatly indebted to Professor Lehto and the reviewer who pointed out to me the Theorem of Gehring and Pommerenke.

\section{Proof of Theorem 3 and its corollaries}

Since $f^{\prime}=-1 / w_{2}^{2}$ and $g^{\prime}=(1 / f)^{\prime}=1 / w_{1}^{2}$, Theorem 1 gives in both cases $\left|f^{\prime}(z)\right| \leqq 1 / v(|z|)$ and $\left|g^{\prime}(z)\right| \leqq 1 / v(|z|)$, respectively, and (41) shows that $f$ and $g$ have finite boundary values everywhere. Proceeding as in Duren's book [2], p. 75, we find

$$
\left|f\left(e^{i \theta}\right)-f\left(e^{i \varphi}\right)\right| \leqq \frac{r|\theta-\varphi|}{v(r)}+2 \int_{r}^{1} \frac{d x}{v(x)},
$$

where $r \in[1 / 2,1)$ is arbitrary. The same holds true for $g$ instead of $f$. Setting $r=1-\eta$, where $1 / 2 \geqq \eta \geqq h=|\theta-\varphi|$, Theorem 3 follows from (54).

Proof of Corollary 1. Since

$$
v(x)=\left(\frac{\sin \lambda x}{\lambda}\right)^{2}
$$

solves (15) with $Q(x) \equiv \lambda^{2}$, Theorem 1 gives

$$
\left|f^{\prime}(z)\right| \leqq\left(\frac{\lambda}{\sin \lambda|z|}\right)^{2}
$$

in $\delta \leqq|z|<1$.

Proof of Corollary 2. One verifies easily that

$$
v(x)=x^{2}\left(1-x^{2}\right)^{\delta}
$$

satisfies (15), where $Q$ is given by the right hand side of (46). Thus, Theorem 3 gives

$$
\Omega(h)=O\left(h^{1-\delta}\right)
$$

and Corollary 2 is proved. 
Proof of Corollary 3. By assumption there exists a positive solution of (50). We may assume that $y$ is even and normalized by $y(0)=1$ (otherwise we consider $(y(x)+y(-x)) / 2 y(0))$. Then

$$
v(x)=\left[y(x) \int_{0}^{x} \frac{d t}{y^{2}(t)}\right]^{2}=x^{2}-\frac{Q(0)}{3} x^{4}+\ldots
$$

satisfies

Setting

$$
v v^{\prime \prime}=-2 Q(x) v^{2}+\frac{1}{2} v^{\prime 2}
$$

we find

$$
Y(x)=\int_{0}^{x} \frac{d t}{y^{2}(t)}
$$

$$
\int_{1 / 2}^{x} \frac{d t}{v(t)}=\int_{1 / 2}^{x} \frac{Y^{\prime}(t)}{Y^{2}(t)} d t<\frac{1}{Y(1 / 2)}, \quad \frac{1}{2} \leqq x<1,
$$

and so, by Theorem $3, f$ has continuous boundary values everywhere.

In the case $Q(x)=1 /\left(1-x^{2}\right)^{2}$ we have $y(x)=\sqrt{1-x^{2}}$ and

Thus, Theorem 3 gives

$$
v(x)=\frac{1}{4}\left(1-x^{2}\right)\left(\log \frac{1+x}{1-x}\right)^{2} .
$$

and Corollary 3 is proved.

$$
\Omega(h) \leqq \frac{4}{\left((\log (1 / h))^{2}\right.}+\frac{8}{\log (1 / h)},
$$

\section{References}

[1] Ahlfors, L. V., and G. Weill: A uniqueness theorem for Beltrami equations. - Proc. Amer. Math. Soc. 13, 1962, 975-978.

[2] Duren, P.: Theory of $H^{p}$ spaces. - Academic Press, New York-London, 1970.

[3] Duren, P., and O. Lehto: Schwarzian derivatives and homeomorphic extensions. - Ann. Acad. Sci. Fenn. Ser. A I Math. 477, 1970, 1-11.

[4] Gehring, F. W., and Ch. Pommerenke: On the Nehari univalence criterion. - To appear.

[5] HiLle, E.: Remarks on a paper by Zeev Nehari. - Bull. Amer. Math. Soc. 55, 1949, 552 -553.

[6] Lehto, O., and K. I. ViRTANen: Boundary behaviour and normal meromorphic functions. Acta Math. 97, 1957, 47-65.

[7] Nehari, Z.: The Schwarzian derivative and schlicht functions. - Bull. Amer. Math. Soc. 55, 1949, 545-551.

Universität Karlsruhe

Mathematisches Institut I

Englerstr. 2

D-7500 Karlsruhe

Bundesrepublik Deutschland

Received 23 March 1983 